\title{
Methodological Paper: COVID-19 and Substance Use Disorder: Study Protocol for the International Society of Addiction Medicine Practice and Policy Interest Group Global Survey
}

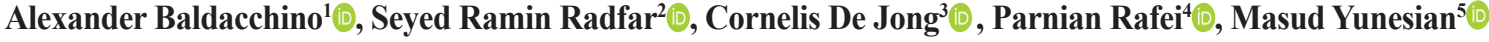

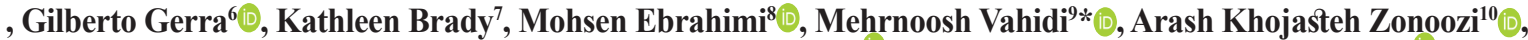 \\ Hossein Mohaddes Ardabili" ${ }^{11}$, Anja Busse ${ }^{12}$, Elizabeth Saenz ${ }^{13}$, Giovanna Campello ${ }^{13}$, Kamran Niaz ${ }^{14}$, Hamed \\ Ekhtiari $^{15}\left[\right.$, Ali Farhoudian ${ }^{16}$ (1)
}

1. Division of Population and Behavioral Sciences, St Andrews University Medical School, University of St Andrews, UK.

2. Integrated Substance Abuse Programs, University of California, Los Angeles, CA, USA.

3. Behavioral Science Institute, Radboud University, The Netherlands.

4. Faculty of Psychology and Education, University of Tehran, Tehran, Iran.

5. Department of Research Methodology and Data Analysis, Institute for Environmental Research, Tehran University of Medical sciences, Tehran, Iran.

6. Drug Prevention and Health Branch, Division for Operations, United Nations Office on Drugs and Crime, Vienna, Austria.

7. Department of Psychiatry and Behavioral Sciences, Medical University of South Carolina, USA.

8. Department of Semiconductors, Materials and Eneroy Research Center (MERC), Tehran, Iran.

9. Department of Psychiatry, Tehran University of Medical Sciences, Tehran, Iran.

10. Student Research Committee, Faculty of Medicine, Mashhad University of Medical Sciences, Mashhad, Iran.

11. Psychiatry and Behavioral Sciences Research Center, Ibn-e-Sina Hospital, Faculty of Medicine, Mashhad University of Medical Sciences, Mashhad, Iran

12. Prevention, Treatment and Rehabilitation Section; Drug Prevention and Health Branch, Division for Operations, United Nations Office on Drugs and Crime, Vienna, Austria.

13. Prevention, Treatment and Rehabilitation Section: Drug Prevention and Health Branch. Division for Operations. United Nations Office on Drugs and Crime, Vienna, Austria.

14. Research and Trend Analysis Branch, Division for Policy Affairs, United Nations Office on Drugs and Crime, Vienna, Austria.

15.Laureate Institute for Brain Research, Tulsa, Oklahoma, USA.

16. Department of Psychiatry, Tehran University of Medical Sciences, Tehran, Iran; Substance Abuse and Dependence Research Center, University of Social Welfare and Rehabilitation Sciences, Tehran, Iran.

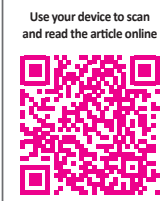

Crtation: Baldacchino, A., Radfar, S. R., De Jong, C., Rafei, P., Yunesian, M., \& Gerra, G., et al., (2020). COVID-19 and Substance Use Disorder: Study Protocol for the International Society of Addiction Medicine Practice and Policy Interest Group Global Survey. Basic and Clinical Neuroscience, 11(2.Covid19), 155-162. http://dx.doi.org/10.32598/bcn.11.covid19.2545.1

http://dx.doi.org/10.32598/bcn.11.covid19.2545.1

(c) (i) 8

Article info:

Received: 05 Apr 2020

First Revision: 06 Apr 2020

Accepted: 09 Apr 2020

Available Online: 15 Apr 2020

Keywords:

COVID-19, SARS-CoV-2, Pandemics, Substance-related disorders, Drug addiction, Public health, Health policy, Disaster medicine, Opiate substitution treatment, Telemedicine, Mental health Services, Health surveys, Country response

\section{AB S T RAC T}

Introduction: As one of the major health problems in the present century, the COVID-19 pandemic affected all parts of the global communities and the health of substance users are potentially at a greater risk of harm. This global study has been designed and conducted by the International Society of Addiction Medicine Practice and Policy Interest Group (ISAM-PPIG) to understand better the health related issues of people with Substance Use Disorders (SUD) as well as responses of the relevant health care systems during the pandemic.

Methods: This is a cross-sectional study using convenient sampling. The data gathering was carried out with two follow-up stages each two months apart through an online conducted survey prepared using Google platform. The survey started by emergence of COVID-19 as a pandemic in March 2020 and respondents were followed till September 2020 when most of the initial lockdowns by most countries are supposed to be reopened.

Ethics and Dissemination: The study was approved by the ethics committee of University of Social Welfare and Rehabilitation Sciences, Tehran, Iran. The results will be published in relevant peer reviewing journals and communicated with different international stakeholders.

* Corresponding Author:

Mehrnoosh Vahidi, MD.

Address: Department of Psychiatry, Tehran University of Medical Sciences, Tehran, Iran.

Tel: +98 (21) 55419151

E-mail: mehrnoosh.vahidi@gmail.com 


\section{Highlights}

- Effects of COVID-19 on PWUD is a major concern for responsible health authorities.

- Effects of COVID-19 on PWUD around the world are different depends on the country.

- This is the first survey that has been conducted internationally after pandemic.

- Results of the survey are essential for further planning in the addiction medicine.

\section{Plain Language Summary}

People who use alcohol or drugs are specifically at stake when facing circumstances like current COVID-19 outbreak for many reasons. More knowledge about the current situation can help us make better plans for this and future similar situations; So, we, as the International Society of Addiction Medicine Practice and Policy Interest Group, aimed to design a study in order to get in touch with experts of addiction medicine in different countries of the world and ask them to share with us what is going on in their countries and how substance users are affected. Also, we aim to know about the responses of related services during the crisis. To do so an online questionnaire was prepared with plenty of questions covering different aspects of addiction and the its management and was circulated among people who are active in this field. After applying statistical analysis, the results will be published in the next articles. Also we are going to repeat the survey each 2 months till 6 months and wish to publish the results as follow-up studies.

\section{Introduction}

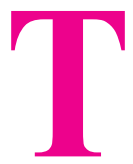

he Coronavirus Disease 2019 (COVID-19) pandemic, is an outbreak of a novel coronavirus pneumonia started in Wuhan Region of China (Zhu et al., 2020). The unique properties of the virus such as its high rate transmission, notable mortality and genetic diversity has created an unprecedented global public health emergency (Phan, 2020). Subsequently on the $11^{\text {th }}$ March 2020, the COVID-19 outbreak was declared a global pandemic by the World Health Organization (WHO) ("Novel Coronavirus emerges in China", 2020). According to the WHO situation report, there were more than 7 million confirmed cases worldwide by $11^{\text {th }}$ June, 2020 (Banerjee, 2020).

COVID-19 pandemic has infected millions of people all around the world. However, it has caused a seismic shift in the billions of people's way of life, especially the vulnerable and high-risk groups. Among the greatest affected communities in periods of such calamities will also include people with Substance Use Disorder (SUD). It logically comes to mind that country-specific restrictions, governmental regulations, quarantine limitations, and lockdowns could have changed the way those people access and use alcohol and drugs. Additionally, these changes would have increased the potential risks of morbidity and mortality (Columb, Hussain,
\& O'Gara, 2020; Kar, Arafat, Sharma, Dixit, Marthoenis \& Kabir 2020). People with SUD are therefore highly challenged by social distancing and health service limitations, especially those who are in treatment or in recovery programs (Volkow, 2020). Regarding those undergoing daily dispensing programs for opioids, missing one single dose of opioid substitution treatment might be enough to instigate a relapse (Farhoudian et al., 2020; Kar et al., 2020). Socioeconomic challenges might make it difficult for homeless people including those with substance use disorders to comply with quarantine measures and might increase their criminal justice contacts.

Reports showed that people with specific socio-demographics (male and old age) and medical conditions (cardiopulmonary diseases, diabetes) are at a greater risk for morbidity and mortality from COVID-19 (Jordan, Adab, \& Cheng, 2020; Li et al., 2020; Parohan, Yaghoubi, Seh raji, Javanbakht, Sarraf, \& Djalali, 2020) On the other hand, we know people with SUD are also at a higher risk for cardiopulmonary complications. The syndemic nature of complex multiple morbidities in people with SUD and additional risks from drug-drug interactions (between COVID-19 medications and the substance which used and/or addiction treatment medications) potentially create a unique situation for people with SUD to experience more complications while dealing with a COVID-19 infection (Farhoudian et al., 2020; Farhoudii an, Nematollahi, Sadeghi, 2020 \& Radfar; Volkow, 2020). 
Additionally, social isolation, chronic quarantine measures, and the pandemic outbreak itself have shown to be associated with increased emotional distress such as depression, anxiety, anger, irritability, and fear (Lai, Shih, Ko, Tang, \& Hsueh, 2020). People are more prone to relapse in these conditions, though experiencing a long-term abstain (Serafini, Toohey, Kiluk, \& Carroll, 2016). Compounding this scenario, group-based effective therapies that were held before the start of the pandemic to support people with SUD with emotional and psychological distress have been canceled due to the restrictions imposed. Switching to internet-based group platforms may not be applicable for all (Columb et al., 2020; Knopf, 2020). Given the above points, addiction medicine is considered as potentially one of the services that could have been severely affected by measures imposed to curb a pandemic such as COVID-19.

In response to this unprecedented situation, the International Society of Addiction Medicine (ISAM) in March 2020 provided:

1. Practical and timely guidelines as a position paper to support practitioners working in addiction medicine during the pandemic (Farhoudian et al., 2020);

2. Four ISAM global webinars, pointing out new raising concerns in the field and some recommendations for managing this overlap of COVID-19 and addiction related responses;

3. Initiation of a global survey which considered as essential as it was well placed to capture unaccounted and unresolved issues and emerging problems emerging in real world situations.

The ongoing changes through different aspects of addiction medicine during different stages of a pandemic, like changes in patterns of drug and alcohol use, price alterations, service access and flexibility, virtual care, education and training qualities raise the need for such a survey. Stemming from the nature of pandemics and their fast and unpredictable alterations, the crucial need for quick situation analysis and responding after is clear. This can be gained by reaching out to experts in the fastest matter of time and collecting their opinions on the existing issues and how the health system is responding to them.

\section{Methods}

\subsection{Study design}

In the present multi-phase, international, cross-sectional study, an online tool was prepared by the research team. Invited participants are key informants and different stakeholders of addiction medicine around the world. The participants were asked to complete the "International Society of Addiction Medicine Practice and Policy Interest Group (ISAM-PPIG) COVID-19 global survey questionnaire".

The first phase of the survey was started on April 4, 2020, in the first week after the WHO's announcement of a pandemic situation and closed on May 7, 2020. After the first phase, the questionnaire will be circulated twice with a 2-month gap each as the 2 follow up phases. The aim is to complete all the data collection by September 2020 from the 3 time points.

\subsection{Participants}

The study participants consisted of nationally-representative informants from different countries who (a) are experts in the field of addiction medicine and health related services, and (b) active in the substance use treatment policy and/or service during the COVID-19 epidemic.

Convenient sampling different databases of relevant e-mail lists were used including:

- ISAM's Board of Directors; ISAM's individuals and affiliate society members;

- Individuals whom the research group had known as the scientists who actively work in the above-mentioned areas;

- Participants of ISAM's global webinars on COVID-19;

- Addiction medicine specialists who actively provide addiction-related services in their countries;

- Individuals who the research group knew them as worldwide policy makers in the area of addiction health care systems.

Since these sources were biased towards North America and Europe, the research team subsequently identified probable participants for each other country through snowballing techniques. As most of these indi- 
viduals were not well-known by the research team, the priorities had been given to those with peer reviewed publications in the area of addiction medicine and related health services. In order to reach these individuals, Pubmed and Google Scholar websites were systematically searched in two serial steps:

\{(heroin [title] OR “opioid drugs" [title] OR opium [title] OR methadone [title] OR cocaine [title] OR methamphetamine [title] OR "amphetamine type stimulants" [title] OR cannabis [title] OR alcohol [title] OR addict* [title] OR "substance use disorder" [title]) AND "health services" OR treatment [title] OR "harm reduction" [title]\} was applied as the first step of search strategy. The first 500 articles' titles were studied and the e-mail addresses and names of corresponding authors were extracted. We removed the authors of the articles that were about basic sciences studies (for instance, genetics, biochemistry, brain imaging, animal studies, molecular, etc.). For the more developed countries we tried to choose the experts with a higher number of articles and citations based on Google Scholar.

A broader search strategy was also conducted with the name of each country in affiliations applied: \{(heroin [title] OR "opioid drugs" [title] OR opium [title] OR methadone [title] OR cocaine [title] OR methamphetamine [title] OR "amphetamine type stimulants" [title] OR cannabis [title] OR alcohol [title] OR addict* [title] OR "substance use disorder" [title]) AND "EACH COUNTRY NAME” [affiliation]\}.

Finally, we looked at social media platforms for relevant scientists and practitioners.

\subsection{Study instrument}

An online tool was prepared using Google platform by the research group. The invitation letter, consent, instructions on how to complete the questionnaire, and the questionnaire itself, all in English. In order to prepare the questionnaire, the following steps were taken:

- A rapid literature review: Literatures and reports showed some concerns and personal views of solutions about people with substance use disorders and their treatment in COVID-19 pandemic circumstances in different regions.

- Expert communication: The research group also communicated with some international experts through ISAM webinars about the related problems in their countries and how they tried to solve them. Their voic- es and slides were gathered and studied by two independent people from the research team to crystallize important messages.

- Reaching a pool of themes: A pool of themes consisting of concerns, problems, advice and solutions was derived from the information gathered and also based on published articles, grey literature and experts' communications.

- Themes categorization: The themes were categorized into "current situation in each country/area", "macrosystem and societal reaction", "service providers' safety", "services continuity, availability and accessibility" "screening and early interventions", "adjustment and flexibility in service", "psychoeducation and psychosocial interventions", "telehealth and online educations", "COVID-19 treatment", and "special populations".

- Making questions: For each category, a few questions based on the above mentioned themes were derived and the responses for each question were organized in an ordering format.

- Adding open-ended question: At the end of each section (a) the participants were asked if there were any important issues that had been missed and (b) asked to rate their countries/areas' reaction toward that concern or problem as a Numeric Rating Scale (NRS) (1-10).

- Revision and qualitative appraisal: The questionnaire was revised several times by the research group. A qualitative appraisal by an expert group consisting of experts in addiction medicine, disaster psychiatry, and epidemiology was asked to refine further the questionnaire.

- Pilot study: We conducted a two-day pilot study with eight individuals and further revisions and editions were applied afterward based on their feedback and comments.

The final questionnaire consisted of 11 sections:

- Situational Assessment

- Managerial and Societal Reaction

- Safety for Service Providers

- Availability and Accessibility of Services

- Screening and Early Interventions 
- Flexibility in Service Provision

- Psychoeducation and Psychosocial Interventions

- Online or Remote Education

- Consultations and other Services

- COVID-19 Service Providers for SUD

- Special and High Risk Populations

After finishing these 11 sections, the participants were asked to describe good practices in their countries. Although not recommended, participants could select "I do not know", "I do not like to answer" and "not applicable" in multiple choice questions, if needed.

In April 2020, different countries were in their different stages of the outbreak and some countries even had not encountered the problem yet. To acknowledge this confounding factor, the team identified the time period that the emergence of COVID-19 was identified per country utilizing the Worldometer website (https:// www.worldometers.info/coronavirus/). Additionally, the severity of the outbreak was recorded in each country as well. These included: 1 . The number of COVID-19 related deaths per capita; and 2. The number of cases that contracted the virus per capita were considered as proxy measures of the severity of the outbreak in each country. Also we searched for developmental status of countries, as reported by the United Nations, and SUD related treatment services available in each country to see if there is any relationship between these variables and the effect of the pandemic on substance use and management of it during a crisis. For the follow up phase of this survey new questions will be added and some will be edited.

\subsection{Data collection procedure}

Informants were invited to participate in the survey by e-mail invitations that were sent via the official email address of the ISAM-PPIG. They were informed of the voluntary nature of the research via the invitation e-mail. However, in order to motivate potential research participants to give exact information, they were asked if they would like to participate as a research team member and their names to be included as an author of the derived articles describing empirical data results. Instructions on purpose, structure, and guidelines for filling out the survey were provided along with the survey link. Also participants were not able to see the answers provided by other people. The link to the questionnaire was also circulated to other expert groups that include addiction medicine specialists such as WADD, EUROPAD, CSAM, ASAM and other affiliated societies of ISAM. The responses were recorded in a private internet database, which was only accessible by the core ISAM-PPIG COVID-19 survey research team. Each participant was allowed to submit one response available on ISAM website (https://isamweb.org/2020/06/isam-practice-and-policy-interestgroup-ppig-global-survey-on-covid-19-and-substanceuse-disorder-sud/).

\subsection{Statistical analysis}

Statistical analyses were conducted according to a multistep plan. The data analyses plan included:

1. Data cleaning of the online dataset with quality control; 2. Descriptive statistics of the general characteristics of the recruited sample, in terms of country of residence, discipline, age, gender, and educational level; 3. Calculation of responses' agreement for each country when there are two or more responses.

Descriptive data were presented as $\mathrm{Mean} \pm \mathrm{SD}$ for each country response, as well as the average of the global responses. Categorical variables for data related for each country were presented as percentages. All statistical analyses were performed using RStudio (V. 1.2.1335). Intraclass correlation coefficient $3 \mathrm{k}$ (ICC $3 \mathrm{k}$ ) analysis was carried out with the aid of 'psych' package (Northwestern University, Evanston, Illinois, USA, http://CRAN.R-project.org/package $=$ psych v. 1.6.4.) to calculate agreement if any between multiple responses recorded from each country or each category of respondents, separately.

This survey was the first of its kind and aimed to evaluate alcohol and substance use related issues during COVID-19 prevalace. Other questionnaires appeared on the similar theme with most using the ISAM-PPIG questionnaire on COVID19 as its template. Additionally, the survey has a global reach and is widely disseminated within the addiction communities in order to get as many participants as possible. It also covered the most important issues that people with substance use may face through a crisis and so the data collected is ecologically relevant.

Limitations were very much based on the need to conduct this survey within the first few weeks of the pandemic. These include the survey (a) not translated into 
other languages, with potential participants withdrawing from the survey and/or some less precise answers, (b) time for participants to complete this survey was not challenging as these same participants were busy dealing with this emerging clinical crisis, (c) the identification of potential participants might have excluded others and the process might have created unintended bias. Finally, methodologically utilizing a survey approach will not collect data that is as accurate as those collected during a multicenter epidemiological studies, however it was considered as an appropriate approach to react as swift as the pandemic.

\subsection{Publication and dissemination strategy}

The results of this survey will be disseminated in (a) peer reviewed publications, (b) discussions with international organizations and (c) within regional policy and practitioner based groups and organizations globally in a timely fashion.

The study aims to explore how different countries in the world responded to the pandemic from a system's, service user and service provider perspectives. The results derived from this worldwide survey will help national and international policy makers to understand the areas that need improved and equally areas that worked well. It will also help prepare policy makers and other stakeholders become proactive in the future in providing the necessary timely and focused response when similar situation arises.

\section{Ethical Considerations}

\section{Compliance with ethical guidelines}

The data was saved on a web database and only the core ISAM-PPIG COVID19 survey research team could access it. Confidentiality of participants was respected. The proposal was submitted to the University of Social Welfare and Rehabilitation Sciences and received the full approval of the ethics committee (Code: IR.USWR.REC.1399.061).

\section{Funding}

The study has been conducted with no funds from external sources.

\section{Authors' contributions}

Alexander Baldacchino, Ali Farhoudian, Seyed Ramin Radfar, Hamed Ekhtiarim and, Cornelis De Jong, Mehrnoosh Vahidi and Parnian Rafei had the original ideal, conducted literature review and designed initial questions and finalized the online version of the questionnaire, and distributed the survey, and participated in analyzing the data; Anja Busse, Elizabeth Saenz, Giovanna Campello and, Kamran Niaz participated in the process of finalizing and circulation of the survey. Masoud Younesian and Mohsen Ebrahimi commented and edited the questions with special guides in statistical analyses and designing the method. Arash Khojasteh Zonoozi, and, Hossein Mohaddes Ardabili participated in the literature review and analyzing of the data and all authors participated in the writing, editing, and revision of the protocol study.

\section{Conflict of interest}

The authors declare that they have no competing interests.

\section{Acknowledgments}

The authors wish to thank Marilyn Dorozio for her help and contributions in this research.

\section{References}

Banerjee, D. (2020). The COVID-19 outbreak: Crucial role the psychiatrists can play. Asian Journal of Psychiatry, 50, 102014. [DOI:10.1016/j.ajp.2020.102014] [PMID] [PMCID]

Columb, D., Hussain, R., \& O'Gara, C. (2020). Addiction Psychiatry and COVID-19-Impact on patients and service provision. Irish Journal of Psychological Medicine, 1-15. [DOI:10.1017/ipm.2020.5] [PMCID]

Farhoudian, A., Baldacchino, A., Clark, N., Gerra, G., Ekhtiari, H., \& Dom, G., et al., (2020). COVID-19 and Substance Use Disorders: Recommendations to a Comprehensive Healthcare Response. An International Society of Addiction Medicine (ISAM) Practice and Policy Interest Group position paper. Autonomic Neuroscience: Basic \& Clinical, 11(2), 129-46. [DOI:10.32598/bcn.11.covid19.1]

Farhoudian, A., Nematollahi, P., Sadeghi, M., \& Radfar, S. R. (2020). Possible Overlap of Laboratory Findings Between Patients with COVID-19 and Substance Use Disorders. Archives of Clinical Infectious Diseases, 15(2). [DOI:10.5812/archh cid.103136]

Jordan, R. E., Adab, P., \& Cheng, K. (2020). Covid-19: risk factors for severe disease and death. British Medical Journal Publishing Group, 368, m1198. [DOI:10.1136/bmj.m1198] [PMID]

Kar, S. K., Arafat, S. Y., Sharma, P., Dixit, A., Marthoenis, M. \& Kabir, R. (2020). COVID-19 Pandemic and addiction: Current problems and future concerns. Asian Journal of Psychiatry. [DOI:10.1016/j.ajp.2020.102064]

Knopf, A. (2020). Addiction telemedicine comes into its own with COVID-19. Alcoholism \& Drug Abuse Weekly, 32(13), 5-6. [DOI:10.1002/adaw.32718] 
Lai, C. C., Shih, T. P., Ko, W.C., Tang, H.-J., \& Hsueh, P. R. (2020). Severe acute respiratory syndrome coronavirus 2 (SARS-CoV-2) and Coronavirus Disease-2019 (COVID-19): The epidemic and the challenges. International Journal of Antimicrobial Agents, 55(3), 105924. [DOI:10.1016/j.ijantimii cag.2020.105924] [PMID] [PMCID]

Li, X., Xu, S., Yu, M., Wang, K., Tao, Y., Zhou, Y., et al., (2020). Risk factors for severity and mortality in adult COVID-19 inpatients in Wuhan. Journal of Allergy and Clinical Immunology. [DOI:10.1016/j.jaci.2020.04.006] [PMCID]

Novel coronavirus emerges in China. (2020). Retrieved from https:/ / www.euro.who.int/en/health-topics/healthemergencies/coronavirus-covid-19/news/news/2020/01/ novel-coronavirus-emerges-in-china

Parohan, M., Yaghoubi, S., Seraji, A., Javanbakht, M. H., Sarraf, P., \& Djalali, M. (2020). Risk factors for mortality in patients with Coronavirus disease 2019 (COVID-19) infection: a systematic review and meta-analysis of observational studies. The Aging Male, 1-9. [DOI:10.1080/13685538.2020.1774748] [PMID]

Phan, T. (2020). Genetic diversity and evolution of SARSCoV-2. Infection, Genetics and Evolution, 81, 104260. [DOI:10.1016/j.meegid.2020.104260] [PMID] [PMCID]

Serafini, K., Toohey, M. J., Kiluk, B. D., \& Carroll, K. M. (2016). Anger and its association with substance use treatment outcomes in a sample of adolescents. Journal Of Child $\mathcal{E}$ Adolescent Substance Abuse, 25(5), 391-8. [DOI:10.1080/106782 8X.2015.1049394] [PMID] [PMCID]

Volkow, N. D. (2020). Collision of the COVID-19 and addiction epidemics. Annals of Internal Medicine. [DOI:10.7326/ M20-1212] [PMID] [PMCID]

Zhu, N., Zhang, D., Wang, W., Li, X., Yang, B., \& Song, J., et al. (2020). A novel coronavirus from patients with pneumonia in China, 2019. New England Journal of Medicine. [DOI:10.1056/NEJMoa2001017] [PMCID] 
This Page Intentionally Left Blank 